\title{
ON COMPACT COMPLEX COSET SPACES OF REDUCTIVE LIE GROUPS
}

\author{
JUN-ICHI HANO ${ }^{1}$
}

1. The statement of theorems. Let $G$ be a connected complex Lie group and let $B$ be a closed complex Lie subgroup in $G$. The left coset space $G / B$ is a complex manifold, which will be called a complex coset space. We denote by $B_{0}$ the identity connected component of $B$ and $U$ the normalizer of $B_{0}$. The canonical projection $p$ of $G / B$ onto $G / U$ defines a holomorphic fibre bundle, and the complex Lie group $U / B_{0}$ acts on $G / B$ as the structure group. We denote by $(G / B, p, G / U)$ this holomorphic fibre bundle.

Suppose that the complex coset space $G / B$ is compact. Then, by a recent result of Borel-Remmert [1, Satz $\left.7^{\prime}\right]$, it turns out that the base space $G / U$ is a Kaehler $C$-space, that is, a simply connected compact complex coset space admitting a Kaehler metric, such that the group of isometries is transitive on it. Since $G / U$ is simply connected, $U$ must be connected. The complex coset space of the connected complex Lie group $U / B_{0}$ by the discrete subgroup $B / B_{0}$ can be regarded as the standard fibre of $(G / B, p, G / U)$. Making use of this result of Borel-Remmert we derive the following.

THEOREM 1. Let $G / B$ be a compact connected complex coset space of a connected complex Lie group $G$ by a closed complex Lie subgroup $B$. Let $U$ be the normalizer of the identity connected component $B_{0}$ of $B$. If $G$ is a reductive complex Lie group, then the fibre of the holomorphic fibre bundle $(G / B, p, G / U)$ is a compact connected complex coset space of a reductive complex Lie group $U / B_{0}$ by the discrete subgroup $B / B_{0}$.

If a compact coset space of a connected reductive real Lie group $G^{\prime}$ by a closed Lie subgroup $B^{\prime}$ admits an invariant complex structure, the complex manifold $G^{\prime} / B^{\prime}$ can be written as a complex coset space of a connected complex reductive Lie group. Hence, this is a case where we can apply the above theorem. This gives a generalization of a theorem proved by Matsushima [2, Theorem 2].

Let $M$ be a connected compact complex manifold. By a theorem of Bochner-Montgomery, the group of all holomorphic homeomorphisms of $M$ onto itself is a complex Lie group acting on $M$ as a holomorphic transformation group. We denote by $A_{0}(M)$ the identity

Presented to the Society January 27, 1963; received by the editors November 20, 1962.

1 Partial support by National Science Foundation Grant No. GP-89. 
connected component of this complex Lie group. If the group $A_{0}(M)$ acts on $M$ transitively, $M$ can be expressed as a complex coset space of a connected complex Lie group $A_{0}(M)$.

TheOREM 2. Let $M$ be a connected compact coset space, and let $A_{0}(M)$ be the identity connected component of the complex Lie group of holomorphic homeomorphisms of $M$ onto itself. If a connected reductive (real or complex) Lie subgroup in $A_{0}(M)$ is transitive on $M$, then $A_{0}(M)$ is a complex reductive Lie group.

When $M$ is a $C$-space, that is, a simply connected compact complex coset space, the fact that $A_{0}(M)$ is a reductive complex Lie group, more strongly that, it is locally a direct product of a complex vector group and a connected semi-simple complex Lie group, is proved by Wang [3, Theorem III].

2. The proof of Theorem 1. We shall prove Theorem 1 in a slightly more general form, which is required in the proof of Theorem 2. Using the same notations as in Theorem 1, let $X$ be a closed complex subgroup in $G$ such that $B \subset X \subset U$. We shall show that if the complex coset space $G / X$ is a Kaehler $C$-space, then the factor group $X / B_{0}$ is reductive. By the theorem of Borel-Remmert mentioned above, the subgroup $U$ satisfies the hypothesis for the group $X$.

Wang's structure theorem asserts that a complex coset space of a connected complex Lie group $G$ by a closed complex Lie subgroup $X$ is a Kaehler $C$-space if and only if $X$ is connected and contains a maximal connected solvable Lie subgroup in $G$ [3]. Therefore, $X$ is connected and contains the identity connected component $Z$ of the center of $G$. We denote by $g$ the Lie algebra of $G$ and by $u, x, b$, and $z$ the subalgebras in $g$ corresponding to the complex Lie subgroups $U, X, B_{0}$, and $Z$, respectively. We always understand that the base field of those Lie algebras is the field of complex numbers. As $G$ is reductive, $g$ is the direct sum of the center $z$ and the maximal semisimple ideal $g_{1}$. We review here a proposition by Wang [3, Proposition 5.2] about the subalgebra $x$ corresponding to the isotropy subgroup $X$ of the Kaehler $C$-space $G / X$. We can choose a Cartan subalgebra $h$ of $g_{1}$ which is contained in $x$, an ordering of the set of roots with respect to $h$, and a subset $\Delta^{\prime}$ consisting of some positive roots, so that a root vector $X_{\alpha}$ belonging to a root $\alpha$ is contained in $x$ if and only if either $\alpha$ is positive or $-\alpha$ is in $\Delta^{\prime}$. Let $\Delta^{\prime \prime}$ be the set of the positive roots not contained in $\Delta^{\prime}$. Then, we have

$$
x=z+h+\sum_{ \pm \alpha \in \Delta^{\prime}}\left\{X_{\alpha}\right\}+\sum_{\beta \in \Delta^{\prime \prime}}\left\{X_{\beta}\right\} .
$$


The subspace $n$ spanned by the $X_{\beta}, \beta \in \Delta^{\prime \prime}$, is a nilpotent ideal in $x$, and a factor algebra of $x$ by an ideal containing $n$ is always a reductive Lie algebra.

Thus, in order to complete the proof of the statement, it suffices to show that $b \supset n$. Since $b$ is an ideal in $x, x$ is stable by ad $H, H \in h$. This implies that $b$ is spanned by $b \cap(z+h)$ and some root vectors. First, we shall show that for a root $\alpha$ in $\Delta^{\prime}, X_{\alpha}$ belongs to $b$ if and only if $X_{-\alpha}$ belongs to $b$. Take a root vector $X_{\alpha}$ such that $X_{\alpha} \in b$ and that either $\alpha$ or $-\alpha$ belongs to $\Delta^{\prime}$. Then, $\left[X_{-\alpha}, X_{\alpha}\right] \in b \cap h$ and $\left[\left[X_{-\alpha}, X_{\alpha}\right], X_{-\alpha}\right]=\alpha\left(\left[X_{-\alpha}, X_{\alpha}\right]\right) \cdot X_{-\alpha} \in b$. As is known in the theory of a semi-simple complex Lie algebra, the complex number $\alpha\left(\left[X_{-\alpha}, X_{\alpha}\right]\right)$ never vanishes, which implies that $X_{-\alpha} \in b$. Let us denote by $\operatorname{ad}_{b} H$ and $\operatorname{ad}_{x} H$ the restrictions of ad $H$ to the subspaces $b$ and $x$, respectively, and by $\bar{\Delta}$ the set of the roots $\alpha$ such that $\alpha \in \Delta^{\prime \prime}$ and that $X_{\alpha} \in b$. Then, from the fact shown above it follows that for any $H \in h$,

$$
\text { trace }\left(\operatorname{ad}_{x} H\right)-\operatorname{trace}\left(\operatorname{ad}_{b} H\right)=\sum_{\alpha \in \bar{\Delta}} \alpha(H) .
$$

Since the coset space $X / B$ of $X / B_{0}$ by the discrete subgroup $B / B_{0}$ is compact, the factor group $X / B_{0}$ is a unimodular Lie group. Therefore, the trace of the linear transformation ad $H, H \in h$, must vanish, and accordingly the sum of all roots in $\bar{\Delta}$ is equal to zero. On the other hand, as every root in $\bar{\Delta}$ is positive, we see that the set $\bar{\Delta}$ is empty. This implies that $b$ contains $n$, completing the proof.

3. The proof of Theorem 2. We denote by $\bar{G}, \bar{B}$ the group $A_{0}(M)$, its isotropy subgroup at a point in $M$, respectively. Let $\bar{U}$ be the normalizer of the identity component $\bar{B}_{0}$ of $\bar{B}$. In virtue of the theorem of Borel-Remmert mentioned above, $\bar{G} / \bar{U}$ is a Kaehler $C$-space and $\bar{U}$ is connected. We denote by $\bar{D}$ the canonical projection of $\bar{G} / \bar{B}$ onto $\bar{G} / \bar{U}$.

Let $G$ be the least connected complex Lie subgroup in $\bar{G}$ containing the given connected reductive Lie subgroup which is transitive on $\bar{G} / \bar{B}$. Obviously, $G$ is also reductive and is transitive on $\bar{G} / \bar{B}$. The isotropy subgroup $B$ in $G$ is $G \cap \bar{B}$. The group $G$ acts on $\bar{G} / \bar{U}$ transitively and its isotropy subgroup $X$ is equal to $G \cap \bar{U}$. Since $\bar{U}$ is the normalizer of $\bar{B}_{0}, X$ is contained in the normalizer $U$ in $G$ of the identity connected component $B_{0}$ of $B$. The complex coset space $G / X$, which coincides with $\bar{G} / \bar{U}$, is a Kaehler $C$-space. By Theorem 1 , the factor group $X / B_{0}$ is a connected reductive complex Lie group. The fibre $\bar{U} / \bar{B}$ of the holomorphic fibre bundle $(\bar{G} / \bar{B}, p, \bar{G} / \bar{U})$ is equal to $X / B$; indeed, we have a holomorphic homeomorphism of $X / B$ onto 
$\bar{U} / \bar{B}$, which is induced from the injection of $X$ into $\bar{U}$. Moreover, we see that the homomorphism of $X / B_{0}$ into $\bar{U} / \bar{B}_{0}$ induced from the injection of $X$ into $\bar{U}$ is locally isomorphic and onto. This follows from the fact that the dimensions of $X / B_{0}$ and $\bar{U} / \bar{B}_{0}$ are equal to those of $X / B$ and $\bar{U} / \bar{B}$, respectively. Thus, $\bar{U} / \bar{B}_{0}$ is a reductive complex Lie group.

Next, we shall show that the dimension of the center of $\bar{G}$ is larger than or equal to that of the center of $\bar{U} / \bar{B}_{0}$. We regard $\bar{U} / \bar{B}_{0}$ as the structure group of the fibre bundle $(\bar{G} / \bar{B}, \bar{D}, \bar{G} / \bar{U})$. Then, the associated principal bundle is $\left(\bar{G} / \bar{B}_{0}, \bar{q}, \bar{G} / \bar{U}\right)$, where $\bar{q}$ denotes the canonical projection from $\bar{G} / \bar{B}_{0}$ onto $\bar{G} / \bar{U}$. Let $\tilde{u}$ be a coset in $\bar{U} / \bar{B}_{0}$, and let $u$ be a representative of the coset $\tilde{u}$. The holomorphic homeomorphism defined by $g \cdot \bar{B}_{0} \rightarrow g u \cdot \bar{B}_{0}, g \in \bar{G}$, is determined by the coset $\bar{u}$, and is the right translation of the principal bundle $\bar{G} / \bar{B}_{0}$ corresponding to the element $\tilde{u}$ of the structure group. Evidently, the right translation commutes with the mapping $g \cdot \bar{B}_{0} \rightarrow x g \cdot \bar{B}_{0}, g \in \bar{G}$, assigned to an element $x$ in $\bar{G}$. We denote by $Z$ the identity connected component of the center of $\bar{U} / \bar{B}_{0}$, and by $n$ the complex dimension of $Z$. The complex Lie group $Z$, being a Lie subgroup in the structure group, acts on $\bar{G} / \bar{B}_{0}$ as a holomorphic transformation group. Let $X_{1}, \cdots, X_{n}$ be linearly independent holomorphic vector fields induced by one-parameter subgroups in $Z$. Then, at each point, they are linearly independent and form a base of the complex tangent space of the orbit of $Z$ through the point. Moreover, each $X_{i}$ is invariant by $\bar{G}$.

Denoting by $s$ the canonical projection of $\bar{G} / \bar{B}_{0}$ onto $\bar{G} / \bar{B}$, we obtain a holomorphic principal fibre bundle $\left(\bar{G} / \bar{B}_{0}, s, \bar{G} / \bar{B}\right)$ whose structure group is the discrete subgroup $\bar{B} / \bar{B}_{0}$ in $\bar{U} / \bar{B}_{0}$. Since $Z$ is in the center of $\bar{U} / \bar{B}_{0}$, each of the holomorphic vector fields $X_{1}, \cdots, X_{n}$ is invariant by the action of the structure group $\bar{B} / \bar{B}_{0}$, and hence they are projectable. Let $Y_{1}, \cdots, Y_{n}$ be their image by the projection $s$. As $s$ is a local homeomorphism, $Y_{1}, \cdots, Y_{n}$ are linearly independent at each point. It is also obvious that each $Y_{i}$ is invariant by $\bar{G}$. The complex manifold $\bar{G} / \bar{B}$ being compact, the holomorphic vector fields $Y_{1}, \cdots, Y_{n}$ generate a connected complex Lie subgroup $\bar{Z}$ of dimension $n$ in $A_{0}(M)$. As $A_{0}(M)=\bar{G}, \bar{Z}$ is in the center of $\bar{G}$ and evidently in the radical $\bar{R}$ of $\bar{G}$.

In order to complete the proof, it suffices to show that the radical $\bar{R}$ of $\bar{G}$ is contained in the center of $\bar{G}$. Since $\bar{G} / \bar{U}$ is a Kaehler $C$-space, $\bar{R}$ is contained in $\bar{U}$, and so is $\bar{Z}$. First, we shall see that the image of $\bar{Z} \cdot \bar{B}$ under the canonical homomorphism $\tau: \bar{U} \rightarrow \bar{U} / \bar{B}_{0}$ contains $Z$. For this purpose, we recall how the group $\bar{Z}$ is constructed. Take an 
element $z$ in $\tau^{-1}(Z)$. To the right translation $f_{\tau(z)}: g \cdot \bar{B}_{0} \rightarrow g z \cdot \bar{B}_{0}, g \in \bar{G}$, of the principal bundle $\bar{G} / \bar{B}_{0}$, there corresponds a holomorphic homeomorphism $g_{\tau(z)}$ of $\bar{G} / \bar{B}$ onto itself, such that $s \cdot f_{\tau(z)}=g_{\tau(z)} \cdot s$. Hence, $g_{\tau(z)}$ is the mapping $g \cdot \bar{B} \rightarrow g z \cdot \bar{B}, g \in \bar{G}$. On the other hand, $g_{\tau(z)}$ is realized by a mapping $g \cdot \bar{B} \rightarrow z^{\prime} g \cdot \bar{B}, g \in \bar{G}$, for a certain element $z^{\prime}$ in $\bar{Z}$. Therefore, we have $z \in z^{\prime} \cdot \bar{B}$, and $Z \subset \tau(\bar{Z} \cdot \bar{B})$. Since the image of $\bar{R}$ under $\tau$ is in $Z$, we have $\bar{R} \subset \bar{Z} \cdot \bar{B}$. It follows that the orbit of $\bar{R}$ through a point is equal to the orbit of $\bar{Z}$; in fact, $\bar{R} \cdot g \cdot \bar{B}=g \cdot \bar{R} \cdot \bar{B}$ $=g \cdot \bar{Z} \cdot \bar{B}$ for any $g \in \bar{G}$. Let $Y$ be a holomorphic vector field induced by a one-parameter subgroup in $\bar{R}$. Then, $Y$ is expressed as a linear combination of $Y_{1}, \cdots, Y_{n}$ whose coefficients are holomorphic functions on $\bar{G} / \bar{B}$. Since $\bar{G} / \bar{B}$ is compact, all the coefficients must be constant. Thus, we have seen that the radical $\bar{R}$ coincides with the central subgroup $\bar{Z}$, and accordingly the dimension of the center of $\bar{U} / \bar{B}_{0}$ is equal to that of the center in $\bar{G}$. From these facts, the assertion of Theorem 2 follows immediately.

REMARK. As an immediate implication of the above theorem, we see that if $M$ is a Kaehler $C$-space, then $A_{0}(M)$ is semi-simple. This is a corollary of a theorem obtained by Matsushima (see Nagoya Math. $\mathrm{J}$. 11). Indeed, $M$ is a complex coset space of a connected complex semi-simple Lie group $G$ by a closed connected complex Lie subgroup $B$, whose normalizer coincides with itself $[3,(5.2)]$. We may assume that $G$ is a subgroup of $A_{0}(M)$. From what we have shown in the above proof, it follows that $\bar{U} / \bar{B}_{0}$ reduces to the identity and accordingly so does the identity connected component of the center of the complex reductive Lie group $A_{0}(M)$. Thus, $A_{0}(M)$ is semi-simple.

\section{BiBLIOGRAPHY}

1. A. Borel and R. Remmert, Über kompact homogene Kählersche Mannigfaltigkeiten, Math. Ann. 145 (1962), 429-439.

2. Y. Matsushima, Sur certaines varietes homogènes complexes, Nagoya Math. J. 18 (1961), 1-12.

3. H. C. Wang, Closed manifolds with homogeneous complex structure, Amer. J. Math. 76 (1954), 1-32.

WASHINGTON UNIVERSITY 